\title{
Impact of the Recent Jefferson Lab Data on the Structure of the Nucleon
}

\author{
W.R.B. de Araújo ${ }^{a}$, T. Frederico ${ }^{a}$, M. Beyer ${ }^{b}$, and H.J. Weber ${ }^{c}$ \\ ${ }^{a}$ Dep. de Física, Instituto Tecnológico de Aeronáutica, Centro Técnico Aeroespacial, \\ 12.228-900 São José dos Campos, São Paulo, Brazil. \\ ${ }^{b}$ Fachbereich Physik, Universität Rostock, 18051 Rostock, Germany \\ ${ }^{c}$ Dept. of Physics, University of Virginia, Charlottesville, VA 22904, U.S.A.
}

Received on 15 August, 2003.

\begin{abstract}
The simultaneous fit of proton ratio $\mu_{p} G_{E p} / G_{M p}, q F_{2 p} / F_{1 p}$ to the recent experimental data and static properties of the nucleon is studied within a light-front model with different spin coupling schemes and wave functions. The position of the zero of proton electric form factor is sensitive to the presence of a hard constituent quark component in the nucleon wave function. The fitting of the new data for the ratios is achieved with a hard momentum scale about 4-5 GeV.
\end{abstract}

\section{Introduction}

The recent $e-p$ polarization transfer experiments performed at the Jefferson Laboratory for square momentum transfers up to $-q^{2} \sim 6 \mathrm{GeV}^{2}[1,2]$, show that the proton ratio $\mu_{p} G_{E p} / G_{M p}$ has a strong and almost linear decrease with $-q^{2}$, which also reveals a flattening of $q F_{2 p} / F_{1 p}$ starting at $-q^{2} \sim 2 \mathrm{GeV}^{2}$. Extrapolation of the linear trend indicates a zero of $G_{E p}$ for $q^{2} \sim 7.7 \mathrm{GeV}^{2}$ [2], which is also incorporated by the new empirical fit of the proton form factors[3].

The constituent light-front model used by us some time ago, with tunable relativistic quark spin coupling forms, expressed in terms of an effective Lagrangian[4], was able to account for the nucleon static properties with pointlike quarks. Although we found a decreasing ratio of $\mu_{p} G_{E p} / G_{M p}$, in qualitative agreement with the experimental data, the momentum transfer at which $G_{E p}$ crosses zero appeared at too low values of $q^{2}$ between 3 to $4 \mathrm{GeV}^{2}$, for different choices of spin coupling scheme and one scale momentum components of the wave function[4]. This model has a too small momentum scale which leads to a zero of $G_{E p}$ at lower values of $q^{2}$. An other light-front model with point-like constituents shows also a zero of $G_{E p}$ at lower values of $-q^{2} \sim 5.5 \mathrm{GeV}^{2}$ [5]. Therefore, one could attempt to introduce another term in the momentum component of the light-front wave function which would bring a higher momentum scale and try to fit the ratio $G_{E p} / G_{M p}$ without changing the conclusions found at low momentum transfers.

In recent works with light-front models[6, 8] applied to mesons, we found the physical motivation to introduce in the nucleon wave function a higher momentum scale related to short ranged physics of the constituent quarks. They found a reasonable description of the meson spectrum and pion properties, including a Dirac-delta interaction in the mass squared operator among other parts, inspired by the hyper- fine interaction from the effective one-gluon-exchange between the constituent quarks[6,7]. It was also pointed out by [9], that the pion and the nucleon light-front wave functions present hard-constituent components, i.e., high momentum tails, above $1 \mathrm{GeV} / \mathrm{c}$, due to the short-range attractive part of the interaction in the spin zero channel, as taken from the Godfrey and Isgur model[10].

The previous discussion physically motivates the presence of a high momentum tail in the valence component of nucleon wave function, which will be tested in calculation of the electromagnetic form factors. In this work, we use a two scale form of the momentum component of the wave function and an effective Lagrangian construction of the spin coupling between the quarks[4], with gradient and scalar forms:

$$
\begin{aligned}
& \mathcal{L}_{\mathrm{N}-3 \mathrm{q}}=\alpha m_{N} \epsilon^{l m n} \bar{\Psi}_{(l)} i \tau_{2} \gamma_{5} \Psi_{(m)}^{C} \bar{\Psi}_{(n)} \Psi_{N}+ \\
& (1-\alpha) \epsilon^{l m n} \bar{\Psi}_{(l)} i \tau_{2} \gamma_{\mu} \gamma_{5} \Psi_{(m)}^{C} \bar{\Psi}_{(n)} i \partial^{\mu} \Psi_{N}+H . C(1)
\end{aligned}
$$

where $\tau_{2}$ is the isospin matrix, the color indices are $\{l, m, n\}$ and $\epsilon^{\operatorname{lm} n}$ is the totally antisymmetric symbol. The conjugate quark field is $\Psi^{C}=C \bar{\Psi}^{\top}$, where $C=i \gamma^{2} \gamma^{0}$ is the charge conjugation matrix. $\alpha$ is a parameter to choose the spin coupling parameterization. The scalar form has $\alpha=0$ and the gradient plus scalar has $\alpha=1 / 2$.

The lower momentum scale of the momentum componet of the light-front wave function is essentially determined by the static observables and the higher one is related to the zero of $G_{E p}$. We choose the harmonic and power-law forms $[11,7]$,

$$
\begin{aligned}
\Psi_{\mathrm{HO}} & =N_{\mathrm{HO}}\left[\exp \left(-M_{0}^{2} / 2 \beta^{2}\right)+\lambda \exp \left(-M_{0}^{2} / 2 \beta_{1}^{2}\right)\right], \\
\Psi_{\text {Power }} & =N_{\text {Power }}\left[\left(1+M_{0}^{2} / \beta^{2}\right)^{-p}+\lambda\left(1+M_{0}^{2} / \beta_{1}^{2}\right)^{-p}\right] .
\end{aligned}
$$


The normalization is determined by the proton charge. The width parameters, i.e., the characteristic momentum scales of the wave function are $\beta$ and $\beta_{1} . M_{0}$ is the free mass of the three-quark system.

This work is organized as follows. In section II, it is given a brief description of the macroscopic and microscopic forms of the nucleon electromagnetic current appropriate for the light-front calculations. In section III, we present the numerical analysis of the nucleon electromagnetic observables for the new two-scale model. A conclusion is presented in section IV.

\section{Nucleon electromagnetic current}

The calculation of the nucleon electromagnetic form factors with the model of Ref.[4], in which the effective Lagrangian from Eq.(1) describes the coupling of the quark spin in the valence component of the wave function, makes use of the plus component of the current $\left(J_{N}^{+}=J_{N}^{0}+J_{N}^{3}\right)$ for momentum transfers satisfying the Drell-Yan condition $q^{+}=$ $q^{0}+q^{3}=0$. The contribution of the Z-diagram is minimized in a Drell-Yan reference frame while the wave function contribution to the current is maximized[12, 14, 15, 7, 16]. We use the Breit-frame, where the four momentum transfer $q=\left(0, \vec{q}_{\perp}, 0\right)$ is such that $\left(q^{+}=0\right)$ and $\vec{q}_{\perp}=\left(q^{1}, q^{2}\right)$, satisfying the Drell-Yan condition.

The nucleon electromagnetic form factors are obtained from the matrix elements of the current $J_{N}^{+}\left(q^{2}\right)$ in the lightfront spinor basis in the Breit-frame constrained by the Drell-Yan condition [17, 4]:

$$
\begin{aligned}
F_{1 N}\left(q^{2}\right) & =\frac{1}{\sqrt{1+\eta}}\left\langle\uparrow\left|J_{N}^{+}\left(q^{2}\right)\right| \uparrow\right\rangle, \\
F_{2 N}\left(q^{2}\right) & =\frac{1}{\sqrt{\eta} \sqrt{1+\eta}}\left\langle\uparrow\left|J_{N}^{+}\left(q^{2}\right)\right| \downarrow\right\rangle,
\end{aligned}
$$

where $F_{1 N}$ and $F_{2 N}$ are the Dirac and Pauli form factors, respectively and $\eta=-q^{2} / 4 m_{N}$. The momentum transfer in the Breit-frame was chosen along the $\mathrm{x}$-direction, i.e., $\vec{q}_{\perp}=\left(\sqrt{-q^{2}}, 0\right)$.

The electric and magnetic form factors (Sachs form factors) are given by:

$$
\begin{aligned}
G_{E N}\left(q^{2}\right) & =F_{1 N}\left(q^{2}\right)+\frac{q^{2}}{4 m_{N}^{2}} F_{2 N}\left(q^{2}\right), \\
G_{M N}\left(q^{2}\right) & =F_{1 N}\left(q^{2}\right)+F_{2 N}\left(q^{2}\right),
\end{aligned}
$$

where $N=n$ or $p$. Here $\mu_{N}=G_{M N}(0)$ and $\kappa_{N}=$ $F_{2 N}(0)$ are the magnetic and anomalous magnetic moments, respectively. The charge mean square radius is $r_{N}^{2}=$ $\left.6 \frac{d G_{E N}\left(q^{2}\right)}{d q^{2}}\right|_{q^{2}=0}$.

Our relativistic model for the nucleon electromagnetic current assumes the dominance of the valence component of the wave function in the static observables and form factors. The microscopic matrix elements of the current are derived from the effective Lagrangian, Eq.(1), within the light-front impulse approximation which is represented by four threedimensional two-loop diagrams [4], which embodies the antisymmetrization of the quark state in the wave function. The detailed form of the expressions used in our calculations were discussed thoroughly in our previous works[4].

\section{Results and Discussion}

The present model of the nucleon light-front wave function has four adjustable parameters: the constituent quark mass, the momentum scales $\beta$ and $\beta_{1}$, and the relative weight $\lambda$ (see Eq.(2)). We use, as before [10, 4, 9], a constituent quark mass value of $m=0.22 \mathrm{GeV}$ in the numerical evaluation of the form factors. The parameters $\beta, \beta_{1}$ and $\lambda$ of the different models are found by reproducing to some extent the proton magnetic momentum and the experimental ratio $\mu_{p} G_{E p} / G_{M p}[1,2]$.

The power-law fall-off from general QCD arguments has a value of $p=3.5$ in Eq.(2)[11, 7]. From the point of view of the static electroweak observables, the value of $p$ does not present an independent feature, once one static observable is fitted the other is strongly correlated, as long as $p>2$ $[4,11]$. Here we choose for our calculations $p=3$. As we are going to show below different assumptions for the wave function, i.e., harmonic or power-law, have minor impact on our conclusions.

We performed calculations with the scalar coupling $\alpha=$ 1 and scalar plus gradient coupling $\alpha=1 / 2$ in the effective Lagrangian, Eq.(1). The last one corresponds to the spin coupling between the quarks to form the nucleon in which the Melosh rotations have the arguments defined by the kinematical momentum of the quarks in the nucleon rest frame. The difference with the Bakajmian-Thomas construction of the spin coupling coefficients resides in the argument of the Melosh rotations which are defined for a system of threefree particles[18]. These subtle differences distinguish the effect of the relativistic spin coupling as an independent feature of the wave function parameterization in respect to the model results for the nucleon form factors[4].

We present results for one-scale and two-scale momentum components of the wave function, for harmonic and power law forms, with $\alpha=0$ and $1 / 2$. The calculations labelled by (a) to (d) and from (e) to (h) in Table I, are done with harmonic and power-law models, respectively. The parameters which we found by a reasonable fit of $\mu_{p}$ and $\mu_{p} G_{E p} / G_{M p}$ simultaneously, are presented in Table I. We observe that while the small momentum scale parameters are about or less than $1 \mathrm{GeV}$, the large momentum scale is found ranging between 4 to $5 \mathrm{GeV}$. The small values of $\lambda$ mean that it is the interference between the two parts of the momentum component of the wave function that carries the high momentum scale in the form factors, and therefore the physical momentum scale which is important for the virtual three-quark system is below $\beta_{1}$ shown in Table I.

The values of the nucleon static observables for all models are shown in Table II. Here, we observe that two-scale models of the wave function produce a reasonable proton 
mean square radius once its magnetic moment is adjusted, as has been found for one scale models which present a strong correlation between these two observables $[11,7,4]$. However, for one scale models the zero of $G_{E p}$ appears at too low momentum transfers with $q_{0}^{2}$ between 3-4 [GeV/c] $]^{2}$, while two-scale models are able to produce a value around 8 $[\mathrm{GeV} / \mathrm{c}]^{2}$. These two-scale models are able to describe reasonably the proton static observables and the zero of $G_{E p}$, as shown in Table II. The neutron magnetic momentum is not reproduced by the models when $\mu_{p}$ is near the experimental value an aspect already found in previous works [4]. Although the neutron charge mean square radius for scalar coupling models agrees with the data, the maximum of the electric form factor is very sensitive to the value of $\mu_{n}$, as we will discuss later.

TABLE I. Parameters of the harmonic and power-law models.

\begin{tabular}{|c|c|c|c|c|c|}
\hline model & type & $\alpha$ & $\beta[\mathrm{MeV}]$ & $\beta_{1}[\mathrm{MeV}]$ & $\lambda$ \\
\hline (a) & $H O$ & 1 & 715 & 5280 & $10^{-4}$ \\
(b) & $H O$ & 1 & 880 & - & - \\
(c) & $H O$ & $1 / 2$ & 726 & 4180 & $1.2 \times 10^{-4}$ \\
(d) & $H O$ & $1 / 2$ & 847 & - & - \\
\hline (e) & Power & 1 & 616 & 5720 & $5 \times 10^{-5}$ \\
(f) & Power & 1 & 1034 & - & - \\
(g) & Power & $1 / 2$ & 660 & 3740 & $10^{-4}$ \\
(h) & Power & $1 / 2$ & 869 & - & - \\
\hline
\end{tabular}

TABLE II. Nucleon electromagnetic static observables and zero of $G_{E p}, q_{0}^{2}$ (in units of $[\mathrm{GeV} / \mathrm{c}]^{2}$ ), for different spin coupling forms with power-law and harmonic models. Experimental values come from ${ }^{*}[19], \dagger[20], \ddagger[21], \S[22]$ and $\downarrow[3]$.

\begin{tabular}{|c|c|c|c|c|c|}
\hline model & $\mu_{p}\left[\mu_{\mathrm{N}}\right]$ & $r_{p}^{2}[\mathrm{fm}]^{2}$ & $\mu_{n}\left[\mu_{\mathrm{N}}\right]$ & $r_{n}^{2}[\mathrm{fm}]^{2}$ & $q_{0}^{2}$ \\
\hline (a) & 2.86 & 0.69 & -1.60 & -0.119 & 8.0 \\
(b) & 2.78 & 0.62 & -1.50 & -0.124 & 3.2 \\
(c) & 2.82 & 0.62 & -1.69 & -0.028 & 8.4 \\
(d) & 2.79 & 0.58 & -1.66 & -0.036 & 3.2 \\
\hline (e) & 2.72 & 0.65 & -1.51 & -0.081 & 7.8 \\
(f) & 2.78 & 0.58 & -1.51 & -0.11 & 4.0 \\
(g) & 2.76 & 0.62 & -1.64 & -0.022 & 8.4 \\
(h) & 2.79 & 0.58 & -1.66 & -0.028 & 4.0 \\
\hline & & $0.66 \pm 0.06^{*}$ & & & \\
Exp. & 2.79 & $0.74 \pm 0.02^{\dagger}$ & -1.91 & $-0.113 \pm 0.005^{\S}$ & $\sim 7.7^{\natural}$ \\
& & $0.77 \pm 0.03^{\ddagger}$ & & & \\
\hline
\end{tabular}
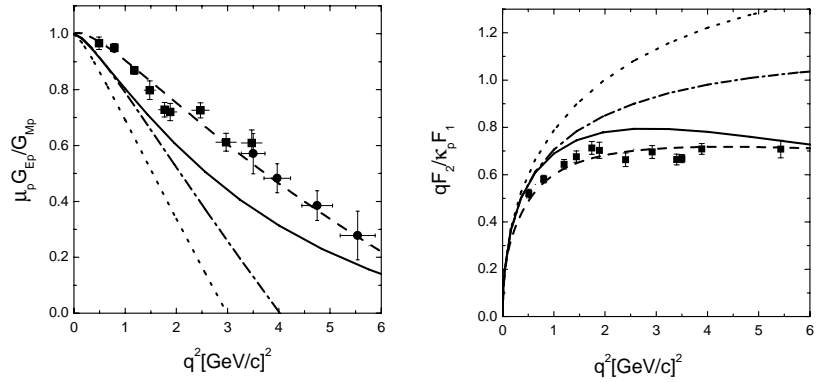

Figure 1. Proton form factor ratios $\mu_{p} G_{E p}\left(q^{2}\right) / G_{M p}\left(q^{2}\right)$ and $q F_{2 p}\left(q^{2}\right) / \kappa_{p} F_{1 p}\left(q^{2}\right)$ for $\alpha=1$. Results for two-scale model (a) (solid line) and (e) (dashed line). Results for one-scale model (b) (dotted line) and (f) (dot-dashed line). Experimental data from Refs. $[1,2]$.

The results for the proton ratios $\mu_{p} G_{E p}\left(q^{2}\right) / G_{M p}\left(q^{2}\right)$ and $q F_{2 p}\left(q^{2}\right) / \kappa_{p} F_{1 p}\left(q^{2}\right)$ using the scalar coupling models, with parameters from Table I, are shown in Fig.1. We present calculations for $\alpha=1$ with harmonic ((a) and (b)) and power-law ((e) and (f)) forms of the wave function. From Table II, one could anticipate the results we found: a reasonable agreement with the data[1,2] is seen for the models which have the zero of $G_{E p}$ around the suggested experimental value of $7.7[\mathrm{GeV} / \mathrm{c}]^{2}$. The same conclusion is found with respect to the ratios when $\alpha=1 / 2$, i.e., the one-scale model adjusted to fit $\mu_{p}$ does not account for the data on the ratios, while two-scale models give a reasonable agreement with the data.

The neutron electric and magnetic form factors for power-law models with $\alpha=1$ ((e) and (f)) and $\alpha=1 / 2$ $((\mathrm{g})$ and $(\mathrm{h}))$ are shown in Fig. 2 . The neutron magnetic moment comes too low, between -1.5 and -1.66 as one see in Table II. Although the neutron mean square radius for scalar coupling is about the experimental value, for one and two-scale models, the peak of $G_{E n}$ strongly depends on the value of $\mu_{n}$. A reasonable agreement with the experimental data for $G_{E n}$ below $5[\mathrm{GeV} / \mathrm{c}]^{2}$ is found when the scalar model is parameterized to fit $\mu_{n}$, while the scalar plus gradi- 
ent model underestimate the data [4]. We found that as $\left|\mu_{n}\right|$ decreases the maximum value of $G_{E n}$ increases. Therefore, the model has to be improved to allow the fit of $\mu_{p}$ and $\mu_{n}$ together. The neutron electric form factor, for both coupling schemes, decreases slowly for two-scale models, due to the presence of the high momentum tail in the wave function, while for the one-scale models it goes faster to zero. In Fig. 2 we also present the calculations for the neutron magnetic form factor, where we found the evidence for a zero for the scalar coupling model with a two-scale wave function. The other parameterizations with one or two-scales models and/or plus gradient coupling does not present a zero up to $100[\mathrm{GeV} / \mathrm{c}]^{2}$. Certainly, the presence or not of the zero in $G_{E n}$ will strongly constrain the models.
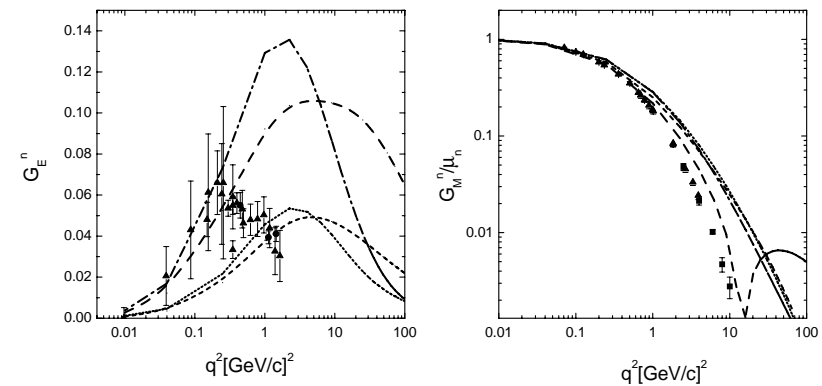

Figure 2. Neutron electromagnetic form factors with power-law models. Results for two-scale model (e) (dashed line) and (g) (short-dashed line). Results for one-scale model (f) (dot-dotted line) and (h) (dotted line). The triangles and the squares are the experimental data from the compilation of Ref.[23] and from Ref.[24], respectively.

\section{Conclusion}

We have overcome the previous limitations of the light-front model to reproduce the nucleon form factors with an effective Lagrangian approach to construct the relativistic spin coupling of the quarks by introducing a physically motivated two-scale wave function model. The zero of the proton ratio $G_{E p} / G_{M p}$ and the static properties, with the exception of the neutron magnetic moment, are reasonably reproduced within a light-front model with scalar spin coupling scheme and harmonic and power-law wave functions. The neutron charge mean square radius is reproduced with the scalar model of the effective Lagrangian, consistent with previous results[4]. The position of the zero is strongly dominated by the presence of a hard constituent quark tail in the nucleon wave function assuming point-like quarks. This result is independent of the detailed form of the quark spin coupling scheme, scalar or scalar plus gradient, and momentum component of the wave function. The present data for the form factor ratios $\mu_{p} G_{E p} / G_{M p}$ and $q F_{2 p} / F_{1 p}$ suggest a hard momentum scale of about $4-5[\mathrm{GeV} / \mathrm{c}]$.

\section{Acknowledgments}

WRBA thanks FAPESP (Fundação de Amparo à Pesquisa do Estado de São Paulo) for financial support, LCCA/USP and CENAPAD/UNICAMP for providing computational facilities. TF thanks CNPq (Conselho Nacional de Pesquisas) and FAPESP.

\section{References}

[1] M.K. Jones et al. [Jefferson Lab Hall A collaboration], Phys. Rev. Lett. 84, 1398 (2000).

[2] O. Gayou et al., Phys. Rev. Lett.88, 092301 (2002).

[3] E.J. Brash, A. Koslov, Sh. Li, and G.M. Huber, Phys. Rev. C65, 051001(R) (2002).

[4] W.R.B. de Araújo, E.F. Suisso, T. Frederico, M. Beyer, and H.J. Weber, Phys. Lett. B478, 86 (2000); E.F. Suisso, W.R.B. de Araújo, T. Frederico, M. Beyer, and H.J. Weber, Nucl. Phys. A694, 351 (2001).

[5] M.R. Frank, B.K. Jennings, and G.A. Miller, Phys. Rev. C54, 920 (1996); G. A. Miller and M.R. Frank, Phys. Rev. C65, 065205 (2002).

[6] H.-C. Pauli, Eur. Phys. J. C7, 289 (1998); "DLCQ and the effective interactions in hadrons" in: New Directions in Quantum Chromodynamics, C.-R. Ji and D.P. Min, Editors, American Institute of Physics, 1999, p.80-139.

[7] S.J. Brodsky, H.-C. Pauli, and S.S. Pinsky, Phys. Rep.301, 299 (1998).

[8] T. Frederico and H. C. Pauli, Phys. Rev. D64, 054007 (2001); T. Frederico, H.C. Pauli, and S.G. Zhou, Phys. Rev. D66, 054007 (2002); Phys. Rev. D66, 116011 (2002).

[9] F. Cardarelli, I.L. Grach, I.M. Narodetskii, E. Pace, G. Salmè, and S. Simula, Phys. Lett. B332, 1 (1994); F. Cardarelli, I.L. Grach, I.M. Narodetskii, G. Salmè and S. Simula, Phys. Lett. B349, 393 (1995); F. Cardarelli; F.Cardarelli, E. Pace, G. Salmè, and S. Simula, Phys. Lett. B357, 267 (1995); Few Body Syst. Suppl. 8 (1995) 345.

[10] S. Godfrey and N. Isgur, Phys. Rev. D32, 189 (1985).

[11] S.J. Brodsky and F. Schlumpf, Phys. Lett. B329, 111 (1994); Prog. Part. Nucl. Phys. 34, 69 (1995).

[12] J. Carbonell, B. Desplanques, V. Karmanov, and J.-F. Mathiot, Phys. Reports 300, 215 (1998), and references therein.

[13] J.H.O. Sales, T. Frederico, B.V. Carlson, and P.U. Sauer, Phys. Rev. C61, 044003 (2000); Phys. Rev. C63, 064003 (2001).

[14] T. Frederico and G.A. Miller, Phys. Rev. D45, 4207 (1992).

[15] J.P.B.C. de Melo, H.W. Naus and T. Frederico, Phys. Rev. C59, 2278 (1999).

[16] B.L.G. Bakker, H.-M. Choi and C.-R. Ji, Phys. Rev. D63, 074014 (2001).

[17] P.L.Chung and F. Coester, Phys. Rev. D44, 229 (1991).

[18] W.R.B. de Araújo, T. Frederico, M. Beyer, and H. J. Weber, J. Phys. G25, 1589 (1999); and references therein.

[19] S. J. Brodsky and J. R. Primack, Ann. Phys. (N.Y.) 52, 315 (1969).

[20] J.J. Murphy II, Y.M. Shin, and D.M. Skopik, Phys. Rev. C9, 3125 (1974).

[21] R. Rosenfelder, Phys. Lett. B479, 381 (2000).

[22] S. Kopecky et al., Phys. Rev. Lett. 74, 2427 (1995).

[23] H. Gao, Int. J. Mod. Phys. E12, 1 (2003); and references therein.

[24] S. Rock et. al., Phys. Rev. Lett. 49, 1139 (1982). 